\title{
Using Google Maps to Generate Organizational Sampling Frames
}

Brad R. Fulton

Indiana University

fulton@indiana.edu

812.855 .6948

1315 E. $10^{\text {th }}$ St.

Bloomington, IN 47405

David P. King

Indiana University

kingdp@iupui.edu

317.278 .8976 


\title{
Using Google Maps to Generate Organizational Sampling Frames
}

\begin{abstract}
Organizational researchers use a variety of methods to obtain sampling frames. The utility of these methods, however, is constrained by access restrictions, limited coverage, prohibitive costs, and cumbersome formats. This article presents a new method for generating sampling frames for any type of organization that is cost-effective, uses publicly available data, and produces near-comprehensive sampling frames for any geographic area in the U.S. The Python-based program we developed systematically scans the Google Maps platform to identify organizations of interest and retrieve their contact information. We demonstrate the program's viability and utility by generating a sampling frame of religious congregations in the U.S. To assess Google Maps' coverage and representativeness of such congregations, we examined two nationally representative samples of congregations and a census of every congregation in Indianapolis. We found that Google Maps contains approximately $98 \%$ of those congregations - near-complete coverage that ensures a nearperfect degree of representativeness. Using Google Maps to generate sampling frames promises to substantially improve the process for obtaining representative samples for organizational studies by reducing costs, increasing efficiency, and providing greater coverage and representativeness.
\end{abstract}

Keywords: organizations, sampling frames, samples, coverage, representativeness, efficiency 


\section{Using Google Maps to Generate Organizational Sampling Frames}

Researchers often seek to examine organizational fields for which there is no easily accessible sampling frame or for which no sampling frame exists. This limitation makes it challenging for organizational scholars to obtain sampling frames that are comprehensive, industry-specific, and affordable. For example, business lists maintained by the Census Bureau and the Bureau of Labor Statistics are difficult to obtain, not organized by industry, and limited to organizations with employees (Bryant \& DeLozier, 1968; Grønbjerg et al., 2010; Jarmin, 2019). In addition, nonprofit organizations with an annual revenue less than $\$ 50,000$ are exempt from filing returns with the Internal Revenue Service (Lampkin \& Boris, 2002), and some organizations (such as religious congregations) are not required to report any information (Scheitle et al., 2016).

Other public records, like telephone directories and U.S. Postal Service databases, are not limited to organizations with employees and may contain low-revenue nonprofits and congregations. However, these records tend to not include transient organizations, and many of the listings are compiled at state and local levels, each constructed independently and with its own unique formatting specifications (Amaya et al., 2014; Murphy, 2002). As a result, merging such databases across levels to create a uniform sampling frame can be very complicated and time-consuming.

Researchers can also use proprietary directories to sample niche industries and hard-toreach fields (Kalleberg et al., 1990) such as high-tech start-ups (Samagaio et al., 2018), microbreweries (Nilsson et al., 2018), and compounding pharmacies (Guharoy et al., 2013). However, obtaining those records is often expensive and few industries have reliable, up-to-date data.

In the absence of an adequate sampling frame, researchers can use hypernetwork sampling to generate a representative sample of organizations (McPherson, 1982). This method works for any type of organization affiliated with individuals, such as hospitals, museums, and unions (McPherson, 2001), and it is commonly used to generate representative samples of business 
organizations (Kalleberg et al., 1994), voluntary associations (Popielarz, 1999), and religious congregations (Chaves et al., 2020; Fulton \& King, 2018). The hypernetwork sampling method, however, has three critical limitations. First, it is time-consuming because it requires two waves of data collection. The first wave surveys a representative sample of individuals and asks them to provide the contact information of an organization they are affiliated with, such as the religious congregation they attend. The second wave surveys the organizations mentioned by the respondents (in this example, the congregations). Second, this method produces only one sample, and the cost of producing that sample increases as the size of the sample increases. Finally, samples generated via hypernetwork sampling also require complex weighting protocols. In particular, the weights must include the weighting for the sample of individuals and account for the likelihood of a particular organization being mentioned by a respondent, which depends on the number of individuals affiliated with that organization (Fulton et al., 2022). ${ }^{1}$ Consequently, the hypernetwork sampling method, although feasible, is neither a viable nor sustainable option for most organizational researchers.

All of the aforementioned sampling frame and sample sources possess significant limitations that undermine their coverage, representativeness, and utility. To address these challenges, we developed a new method for generating organizational sampling frames that compiles publicly available data contained on the Google Maps platform. Google Maps functions as a data repository that gathers information from multiple sources and continually organizes and updates its data. More specifically, Google Maps' "Places” feature obtains and curates information about every organization on its platform. ${ }^{2}$ The program we developed extracts this information to generate

\footnotetext{
${ }^{1}$ The probability that an organization appears in a sample generated by the hypernetwork sampling method is proportional to the number of people affiliated with the organization. Because organizations are nominated by individuals affiliated with them, organizations with more people are more likely to be in the sample than organizations with fewer people. Although larger organizations are overrepresented in the sample, they are overrepresented by a known degree and their overrepresentation can be accounted for with weights.

2 As of February 2023, Google Maps reports that there are over 200 million places on its platform and that 25 million updates to the places' information are made daily (https://cloud.google.com/maps-platform/places).
} 
organizational sampling frames. Our program uses Google Maps' application programming interface (API) to search for particular types of organizations in specific geographic areas and then compiles a list of organizations that meet the search criteria.

To demonstrate the viability and utility of this method, we used the program to generate a sampling frame of religious congregations in the U.S. - a population for which no sampling frame exists (Brauer, 2017; Chaves, 2002). To assess the coverage and representativeness of congregations listed on Google Maps, we used two nationally representative samples of congregations and a census of every congregation in Indianapolis. We found that $98 \%, 97 \%$, and $98 \%$ of the congregations in those samples, respectively, are on Google Maps. We then assessed Google Maps' representativeness by examining characteristics of the congregations not on Google Maps (e.g., size, age, religious tradition, online presence, type of meeting facility, and community setting). Apart from congregations with fewer than 100 members being marginally less likely to be on Google Maps, we found no other characteristic associated with not being on Google Maps. Moreover, Google Maps’ nearcomplete coverage of congregations ensures a near-perfect degree of representativeness. ${ }^{3}$

Overall, using Google Maps to generate sampling frames is superior to all other existing methods. Compared to using data from public records, Google Maps has greater coverage and returns the data in one consistent format. Unlike methods that use data from proprietary records, Google Maps' data include a broader range of organization types and cost less to obtain (Simsek \& Veiga, 2001). Finally, compared to the hypernetwork sampling method, our method is less expensive and more efficient, can provide multiple samples and generate larger samples without increasing costs, and does not require complex weighting protocols.

\footnotetext{
${ }^{3}$ Complete coverage means that the sampling frame contains every unit in the target population (i.e., no undercoverage). Perfect coverage means that the sampling frame has complete coverage and includes no out-of-scope units (i.e., no overcoverage). Perfect representativeness means that no subunits are over- or under-represented in the sampling frame.
} 
Our method can be applied to any type of organization across any geographic area and is particularly beneficial for organizations for which a sampling frame is expensive, not easily accessible, or does not exist. In addition to producing a comprehensive sampling frame from which random samples can be drawn, researchers can use this method to estimate the total number of organizations in a particular geographic area, conduct geospatial analyses, and test organizational ecology theories. In short, using Google Maps to generate sampling frames promises to significantly improve the process for obtaining representative samples for organizational studies by reducing costs, increasing efficiency, and providing greater coverage and representativeness.

\section{Generating Sampling Frames from Google Maps}

In this section, we describe the Python-based program we developed to generate sampling frames by extracting data from the Google Maps platform on particular organizations of interest within specified geographic areas. ${ }^{4}$ The program uses the Google Maps Places API, a service researchers can access using a software program that returns requested data from the platform in a structured uniform format ready to analyze (see Appendix A for links to the Google Maps Place API features mentioned in this article).

\section{Google Maps Places}

Google Maps categorizes organizations as "places" and assigns each organization a unique place ID. Then, it assigns each organization a place type (selected from a list of 97 place types) that broadly describes the organization's type. The list includes terms such as school, hospital, and supermarket (see Appendix B for the complete list). In addition, each place (organization) can be assigned up to 10 business categories that provide more specific details. The list of 4,050 business categories includes terms such as charter school, animal hospital, and Asian grocery store. ${ }^{5}$

\footnotetext{
${ }^{4}$ After the anonymous peer-review process concludes and a journal decides to publish this manuscript, the authors will make the code for this program available to researchers.

${ }^{5}$ The complete list of business categories can be viewed at https://pleper.com/index.php?do=tools\&sdo=gmb_categories
} 
Google Maps obtains information on places from multiple sources. Specifically, Google collects data on organizations from their websites, online postings, and public records. In addition, owners can list their organization as a place on Google Maps by registering it at Google My Business, and internet users can manually add an organization or update its information on Google Maps. Although this feature is named "Google My Business," any organizational entity—business, nonprofit organization, or public institution — can be registered on Google Maps using this feature. The platform consolidates the information on each organization and stores it as place details. As a result, compared to other sampling frames, Google Maps can incorporate new organizations more quickly as well as organizations that do not have a fixed location.

Regrettably, Google Maps does not provide a list of all the places (i.e., organizations) on its platform. If such a list was available, researchers could simply draw samples from that list (Braun et al., 2018). Instead, Google offers the Google Maps Places API service that researchers can use to search for places of interest on Google Maps and request data on those places.

\section{Finding Organizations and Obtaining Organizational Data from Google Maps}

The program we developed finds organizations on the Google Maps platform using the API's Place Search request, which returns a list of places based on a specific search request and corresponding search criteria. Our program specifically uses the Nearby Search option because it allows researchers to search for a particular type of place within a specified geographic area. This search request returns the place IDs of organizations that meet the search criteria. Using the place IDs, the program then uses the Place Details request to obtain information about the organizations, including their contact information. Each organization's name and corresponding data are added to the sampling frame database.

To ensure that the search includes the entire specified geographic area, the program uses a raster tile mapping schema that divides the world into small square tiles, each with a fixed geographic 
area and scale that corresponds with the assigned zoom level (Stefanakis, 2017). For example, a map at zoom level 15 creates square tiles that each represent 1.495 square kilometers (approximately 1 square mile), and those tiles form a two-dimensional array that covers the geographic area of interest. $^{6}$

Our program begins by setting the geographic boundaries of the search area. It starts at zoom level 15 and creates a two-dimensional array of tiles that covers the geographic area. The program then selects the first tile in the array and sets the query point at the center of that tile. The API's Nearby Search request allows researchers to specify the radius of the query area. For a circular query area to encompass the entire area of a square tile, the radius parameter is set to $l(\sqrt{2}) / 2$ where $l$ is the length of the tile. Because a portion of the query area extends beyond the boundaries of a tile, the query areas of adjacent tiles will overlap, and organizations in the overlapping part of the query areas will be returned by more than one search request. Knowing this possibility, we designed the program to reject a returned organization if it contains the same place ID as an organization already stored in the database.

A constraint of the Google Maps Places API is that it will return no more than 60 organizations with each search request. Our program accommodates this constraint by dynamically adjusting the size of the tile and radius of the query area. If a search request returns fewer than 60 organizations, then the search result contains all the organizations of interest from within that query area. If the search request returns exactly 60 organizations, it means the query area contains 60 or more organizations of interest. In such cases, the program deletes the results of that search request and divides the tile into four equally-sized square tiles, each of which is .374 square kilometers (zoom level 16). The program repeats the query process with each of these four tiles-selecting a query

\footnotetext{
${ }^{6}$ The zoom levels on Google Maps range from 1 (zoomed all the way out), where the entire world is contained on one tile, to 20 (zoomed all the way in), where each tile is 1,461 square meters (equivalent to a mid-sized building).
} 
point, setting the radius of the query area, and submitting a search request. If the search request returns fewer than 60 organizations, the program moves to the next tile. If the search request returns exactly 60 organizations again, it repeats the process of deleting the results and dividing the tile into fourths. These steps repeat for each tile until the search request returns fewer than 60 organizations, thus ensuring all the organizations of interest in that tile have been identified. The purpose of this design feature is to confirm that no searched query area contains 60 returned organizations - an outcome where a theoretical $61^{\text {st }}$ organization might not have been returned by the Google Maps Places API. The program continues until every tile has been searched and each tile contains fewer than 60 organizations of interest.

After all the organizations of interest for the specified geographic area have been returned, the program then obtains the contact information for each organization using its place ID and Google Maps' Place Details request. Creating such a database serves two main purposes: 1) to identify the entire population of organizations in a geographic area and estimate the total number of organizations in that area; and 2) to provide a near-comprehensive sampling frame from which random samples can be drawn. See Figure 1 for a visual depiction of the entire process.

[Insert Figure 1 Here]

\section{Generating a Sampling Frame of U.S. Congregations}

To demonstrate our program's functionality and assess its performance, we used it to generate a sampling frame of religious congregations in the U.S. by identifying every congregation on Google Maps. Although we could assess the program using any type of organization, congregations are a fitting test case because they exist in communities throughout the country-from densely populated urban centers to sparse rural areas. The field of congregations also has characteristics similar to other organizational fields. Congregations vary substantially along several dimensions (e.g., size, age, religious tradition, online presence, and type of meeting facility) (Adler et al., 2020). Like other 
organizational types, congregations can operate in a variety venues and multiple congregations can operate out of the same building (King et al., 2019). In addition, the birth and death rates of congregations are similar to those of other organizational populations (Anderson et al., 2008;

Pitt, 2021). These characteristics make congregations an appropriate population for testing the viability and utility of our program.

In the program, as part of the Place Search request, we directed the API to return every place within the U.S. identified by Google Maps as one of the following types: church, synagogue, mosque, or Hindu temple. ${ }^{7}$ The program generated a database of congregations that includes the name and address of each congregation, and when available, its phone number and website address. In the following section we examine the resulting sampling frame of U.S. congregations to assess the coverage and representativeness of congregations on the Google Maps platform.

\section{Assessing Google Maps' Coverage and Representativeness}

The comprehensiveness of places on Google Maps is not known. Specifically, researchers do not know the percentage of organizations on Google Maps nor the characteristics of organizations not on Google Maps. Because most of Google Maps' internal data collection methods are proprietary and the biases associated with the crowdsourcing methods Google Maps uses to update its data are not overt (Bright et al., 2018; Budhathoki \& Haythornthwaite, 2013), the coverage and representativeness of organizational sampling frames generated from Google Maps need to be assessed. Below, we illustrate how to conduct such assessments using representative samples and a census of a particular type of organization on Google Maps.

Sampling frames generated from Google Maps are susceptible to both undercoverage and overcoverage error. Undercovered units are in the target population, but not included in the sampling

\footnotetext{
${ }^{7}$ In almost every case, Google Maps assigns congregations one of these four place types regardless of its religious tradition. For example, it is not uncommon for Google Maps to assign a Buddhist temple the place type "church."
} 
frame, while overcovered units are included in the sampling frame, but are not in the target population (Harter et al., 2016). Undercoverage exists when the sampling frame does not include an eligible organization because it is not listed on Google Maps or because Google Maps miscategorized the organization's type. For example, if Google Maps mistakenly assigns a particular mortuary the place type "cemetery" instead of "funeral home," it would not be included in a sampling frame of funeral homes. Overcoverage exists when the sampling frame includes an organization that is no longer operating or an organization that is out of scope because Google Maps miscategorized its type. For example, if Google Maps mistakenly assigns a particular pet store the place type "zoo" instead of "pet store," it would be included in a sampling frame of zoos. Overcoverage also exists when an eligible organization is listed more than once. For instance, a double listing occurs when an organization moves to a new location and Google Maps registers the new location before purging the old one. In such instances, the organization is listed twice on Google Maps and the sampling frame will contain a duplicate case of that organization.

To assess Google Maps' coverage of congregations throughout the U.S., we examined two nationally representative samples of congregations that were generated via the hypernetwork sampling method. These samples were created for the National Study of Congregations' Economic Practices (NSCEP) (Fulton \& King, 2018) and the National Congregations Study (NCS) (Chaves et al., 2020). Both studies produced their hypernetwork sample of congregations by surveying a nationally representative sample of individuals - the NSCEP used the AmeriSpeak Panel (Dennis, 2019), and the NCS used the General Social Survey (Smith et al., 2019). In both surveys, respondents who indicated attending religious services at least once a year were asked to provide the name and location of their congregation. Researchers then attempted to contact a key informant at each congregation (typically the head clergyperson) and ask them to complete a survey about the congregation. In total, 1,233 congregations participated in the NSCEP and 1,262 congregations 
participated in the NCS. Theoretically, the sampling frames from which the NSCEP and NCS samples were randomly drawn contain all of the congregations in the U.S. enabling us to use them to assess the coverage and representativeness of congregations on the Google Maps platform (Fulton et al., 2022).

Assessing the coverage involves determining the percentage of congregations in the NSCEP and NCS samples that are on Google Maps. Because congregations can have very similar names and more than one congregation can operate out of the same building, we had to search for the congregations on Google Maps manually. As part of this search process, we constructed the binary variable "on Google Maps" that indicates whether we found the congregation on the text layer of Google Maps. ${ }^{8}$ The analysis indicates that $98 \%$ and $97 \%$ of the congregations in the NSCEP and NCS samples are on Google Maps. ${ }^{9}$

To assess Google Maps' coverage of congregations for a specific geographic area, we examined a census of congregations in Indianapolis maintained by the Center for Congregations (CfC). ${ }^{10}$ Of the 937 congregations in Indianapolis, 920 (98\%) are on Google Maps. Analysis of these three independent datasets indicates that most of Google Maps' undercoverage of congregations is due to congregations not being on Google Maps, rather than Google Maps miscategorizing congregations as something other than congregations. Among the 27 congregations not included in the sampling frame, only two were miscategorized—one as a health center and the other as a food bank. The analysis demonstrates Google Maps' near-complete coverage of congregations, and

\footnotetext{
${ }^{8}$ The Google Maps platform has a visual layer and a text layer. Because the search program scans the text on Google Maps, we do not consider a congregation to be "on Google Maps," if only its image appears on the visual layer of Google Maps (i.e., via satellite or street view). We consider a congregation to be on Google Maps only if its name appears on the text layer of Google Maps.

${ }^{9}$ The hypernetwork sampling method used to create the NSCEP and NCS samples yields a probability-proportionalto-size sample (i.e., the probability of a congregation being nominated corresponds with the number of adults that attend its services), which means that larger congregations are more likely than smaller congregations to be included in the samples. All of the analyses account for the overrepresentation of larger congregations by applying congregationlevel weights that undo the probability-proportional-to-size bias in the NSCEP and NCS samples.

${ }^{10} \mathrm{We}$ selected Indianapolis because it is one of the few sufficiently large geographic areas for which a comprehensive database of every religious congregation in the area is maintained and regularly updated.
} 
given Google Maps' multiple modes for acquiring data (Rhodes et al., 2015), its undercoverage error for organizational populations likely will continue to decrease (Tonidandel et al., 2018).

Sampling frames generated from Google Maps are also susceptible to overcoverage error: including organizations more than once or including organizations outside the scope of the sampling frame parameters. We assessed Google Maps' overcoverage of congregations by examining the sampling frame of congregations in Indianapolis generated by our program. Although our program ensures that the sampling frame will not include organizations outside the specified geographic scope, it could include organizations that are no longer operating or organizations that Google Maps miscategorized. Of the 948 records in the Indianapolis sampling frame generated from Google Maps, $10(1.1 \%)$ were no longer operating and $5(0.5 \%)$ were not congregations. We also used the Indianapolis sampling frame to estimate the percentage of organizations listed more than once on Google Maps. Only 13 (1.4\%) of the 948 records were duplicates and no congregation was listed more than twice. ${ }^{11}$

Based on the analysis of congregations in Indianapolis, we estimate Google Maps' undercoverage error to be $2 \%$ and its overcoverage error to be $3 \%$. When researchers purchase a sample, they typically do not have access to the sampling frame - making it impossible to calculate its coverage error (Harter et al., 2016). Even when a sampling frame is available, it is often impractical to identify the erroneously excluded and included records needed to calculate its coverage error (Groves et al., 2009). Thus, for most studies, a frame's undercoverage remains unknown and its overcoverage is not detected until a sample is drawn and analyzed. Compared to studies for which the sampling frame's coverage error is calculated, Google Maps' estimated coverage error is among the lowest (Kölln et al., 2019; Weisberg, 2009), distinct in having both very low undercoverage and

\footnotetext{
${ }^{11}$ Although there are only 937 congregations in Indianapolis, the original sampling frame generated by our program contained 948 records, 28 of which were either out-of-scope or duplicate records. Thus, the final sampling frame contained 920 valid records.
} 
overcoverage error (Harter et al., 2016). These analyses demonstrate that Google Maps’ sampling frame of congregations has near-perfect coverage (i.e., it almost perfectly matches the target population) (Groves et al., 2009), and it is reasonable to expect similar coverage for other types of organizations (Ibarz \& Banerjee, 2017; Schmidt \& Weiser, 2012).

However, a sampling frame can have low coverage error and still not be representative of the target population. To assess the representativeness of congregations on Google Maps, we examined the three datasets (NSCEP, NCS, and CfC) to ascertain whether particular congregational characteristics are associated with not being on Google Maps. Typically, it would be appropriate to conduct logistic regression analyses using the variable "not on Google Maps" as the dependent variable and various congregational characteristics as the independent variables. However, given the rarity of a congregation not being on Google Maps and the corresponding very small number of such cases in the NSCEP, NCS, and CfC samples (4, 6, and 17, respectively), conducting a standard logistic regression or even alternative estimation methods for rare events (e.g., Firth, 1993; Hirji et al., 1987; King \& Zeng, 2001; Tonidandel \& LeBreton, 2010) would not produce reliable results (van Smeden et al., 2019). The very small number of events (i.e., not being on Google Maps) would amplify the small-sample bias known to affect the maximum likelihood estimation of the logistic model and substantially undermine the reliability of the estimates produced by such analyses (Gart \& Zweifel, 1967; Nemes et al., 2009).

As an alternative to conducting logistic regressions, we examined characteristics of the 27 congregations not on Google Maps. We anticipated that the types of congregations less likely to be on Google Maps are new, non-Christian, have minimal online presence, meet in a nontraditional building (e.g., they rent space in a strip mall, school, or community center), are located in a rural setting, and/or have few members. However, examination of the congregations not on Google Maps indicates distributions for the congregations' age and religious affiliation consistent 
with the distributions for congregations in the U.S. In addition, $44 \%$ have a website, $63 \%$ own (versus rent) their building, and $40 \%$ are located in an urban setting; proportions that roughly correspond with the proportions for the entire field of congregations. ${ }^{12}$ Although most of the congregations have fewer than 100 members, the median size of congregations in the U.S. is approximately 50 members (Adler et al., 2020). Thus, apart from having fewer than 100 members, we did not identify any specific characteristic associated with a congregation not being on Google Maps. In summary, the low risk of congregations not being on Google Maps combined with no major differences between eligible congregations on and off the frame, demonstrates the representativeness of congregation sampling frames generated from Google Maps.

\section{Limitations}

Our analysis identified three key limitations that affect the validity of the sampling frames generated from Google Maps. The first limitation concerns overcoverage error: including organizations outside the scope of the sampling frame parameters (ineligible cases). The types of ineligible cases include 1) organizations that are no longer active, but are still listed on Google Maps and 2) organizations that Google Maps has miscategorized as the target population type. For example, the congregation sampling frame we generated contains a school, a foundation, and a fraternal association that Google Maps miscategorized as congregations. Our examination of Google Maps' sampling frame of congregations in Indianapolis indicates an overcoverage error of $1.6 \%$ (10 cases were no longer operating and 5 cases were not congregations).

Although overcoverage will inflate the population count for target populations, researchers can estimate a sampling frame's overcoverage error by drawing a sample and identifying the percentage of ineligible cases it contains. They can then revise the estimated target population size

\footnotetext{
12 The calculation for the percentage of congregations located in an urban setting does not include the congregations from the $\mathrm{CfC}$ dataset, because all of the congregations in that dataset are in an urban setting.
} 
accordingly. For sampling purposes, however, it is not necessary for researchers to search the sampling frame for inactive or miscategorized cases. Instead, if researchers discover an ineligible case among their sample, they can remove it from the sample and replace it with a new case drawn at random from the sampling frame (see Harter et al., 2010:173-4). Alternatively, researchers could address overcoverage by oversampling (Kalton, 2021).

The second limitation concerns duplication error: when multiple cases in a sampling frame are linked to the same organization in the target population (duplicate cases). A sampling frame generated from Google Maps will contain a duplicate case of an organization if the organization has moved and is listed on Google Maps at both its old and new location. Our examination of Google Maps' sampling frame of congregations in Indianapolis indicates a duplication error of 1.4\% (13 cases were duplicates).

Duplicate cases are more concerning because they will inflate the population count for target populations, and they are twice as likely to be selected in random samples drawn from the sampling frame. Researchers can attempt to identify duplicate cases in the sampling frame by flagging cases that have the same name or phone number. However, not all such cases represent duplicates, as it is possible for two unique organizations to have the same name or phone number. For example, they might share a phone line, or both might list the phone number of the same regional office or national headquarters.

When it is not feasible to purge duplicates from the sampling frame prior to sample selection, researchers can address this concern during subsequent data collection. They can include an item in their study that asks respondents whether their organization has moved locations in the past year, and if so, request the address of its previous location. Then, researchers can search the sampling frame for duplicate cases of those organizations. If they discover a duplicate in the sampling frame, they can make the population count more accurate by removing the duplicate; specifically, the case 
with the less-current location information. They can also correct the duplicate case's doubled likelihood of being selected by applying a weight of .5 to the sampled case, thereby reducing by one-half its relative contribution to the sample estimates (see Groves et al. 2009 and Kalton 2021 for other ways to handle such cases). In addition, the duplication error of a sampling frame generated from Google Maps is likely to be very small given that Google Maps works continuously to detect and eliminate duplicate listings (Manasse, 2009).

The third limitation concerns undercoverage error: not including organizations that are in the target population (eligible cases not included in the sampling frame). The types of eligible but erroneously excluded cases include 1) organizations that are not on the text layer of Google Maps (omitted cases) and 2) organizations that Google Maps miscategorized as not being the target population type. For example, the congregation sampling frame we generated does not include a particular congregation because Google Maps miscategorized it as a food bank. Our analysis indicates that Google Maps' sampling frame of congregations has an undercoverage error of approximately $2 \%$, and it is reasonable to expect a similar percentage for other organizations (Ibarz \& Banerjee, 2017; Schmidt \& Weiser, 2012). Furthermore, we expect the percentage of organizations not on Google Maps to decrease over time given Google Maps' multiple modes for populating its database and because it allows users to add organizations to its platform. We also expect Google Maps to increase its accuracy in categorizing organizations as its artificial intelligence and natural language processing algorithms improve (Hickman et al., 2020; Kobayashi et al., 2018), and because it allows users to correct organizations' information on Google Maps.

Undercoverage will create an undercount of the target population. However, researchers can conduct analyses similar to those discussed above to estimate the percentage of eligible organizations that were omitted or miscategorized, and then revise the estimated target population size accordingly. In addition, Google Maps' very low undercoverage error and our analysis of its representativeness 
suggest that undercoverage bias will not significantly impact analyses that use sampling frames generated from Google Maps.

Overall, the limitations associated with using Google Maps to generate sampling frames are minimal, especially compared to the limitations of alternative methods, and can be mitigated through supplemental analyses. In addition, sampling frames generated by this approach can be enhanced by applying it to other online data repositories that obtain their data from a variety of different sources (Bhutta, 2012). For example, Yelp is an online directory of businesses that crowdsources its data from user-supplied information and reviews. Alternatively, YellowPages.com gathers its information on organizations primarily from phone companies and business owners. Furthermore, an increasing number of organizations, such as SafeGraph and Esri, are building open data platforms and compiling places datasets. Although the data on the Google Maps platform are the most comprehensive, using multiple repositories that obtain their data from complementary sources allows for cross-checking and could further increase the coverage and representativeness of the sampling frames that are generated.

Moving forward, by making our program available to researchers will help to improve the quality of the sampling frames it produces. As researchers use Google Maps to generate more sampling frames for more types of organizations and conduct studies on samples drawn from those frames, estimates of overcoverage and undercoverage error will improve and be calculable for multiple types of organizations. Furthermore, coverage error is far from unique to sampling frames generated from Google Maps. Every database is susceptible to this error and its severity is inversely related to how often the databases are updated and the accuracy of their categorizations. For Google Maps, we expect its error rates to continue to decrease as it updates its databases more frequently and increases the accuracy of its categorizations (Ibarz \& Banerjee, 2017; Schmidt \& Weiser, 2012). 


\section{Conclusion}

Most researchers obtain sampling frames from sources such as government records, public databases, and proprietary directories. However, those sources tend to have access restrictions, limited coverage, prohibitive costs, and cumbersome formats. By comparison, our method of generating sampling frames from Google Maps mitigates many of those shortcomings and offers several advantages. It can be applied to any type of organization and is particularly beneficial for organizations where a sampling frame is expensive, not easily accessible, or does not exist. From the sampling frames it produces, researchers can draw multiple samples of varying sizes without increasing cost. In addition, because the method allows researchers to specify the geographic area and search criteria, researchers can save time by not having to identify and remove organizations outside their scope of study.

Furthermore, the information that traditional sampling frame sources contain is often outdated because they are typically updated and published no more than once per year. In contrast, because the information on Google Maps is continuously updated, obtaining sampling frames using this method is not bound by structured reporting and publication schedules. Researchers can run the program at any time and generate a sampling frame with the most up-to-date information available.

In addition to generating sampling frames from which random samples can be drawn, researchers can use this method to estimate the total number of organizations in a particular geographic area and conduct geospatial analyses. Also, by running the program at regular intervals, researchers can obtain more refined measures of field-level changes over time and use these longitudinal data to test organizational ecology theories. In short, using Google Maps to generate sampling frames promises to significantly improve the process for obtaining representative samples for organizational studies by reducing costs, increasing efficiency, and providing greater coverage and representativeness. 


\section{References}

Adler, G. J., Jr., Fulton, B. R., \& Hoegeman, C. (2020). Survey data collection methods and discrepancy in the sociological study of religious congregations. Sociology of Religion, 81(4), 371-412.

Amaya, A., LeClere, F., Fiorio, L., \& English, N. (2014). Improving the utility of the DSF address-based frame through ancillary information. Field Methods, 26(1), 70-86.

Anderson, S. L., Martinez, J. H., Hoegeman, C., Adler, G., \& Chaves, M. (2008, Jun). Dearly departed: How often do congregations close? [Article]. Journal for the Scientific Study of Religion, 47(2), 321-328.

Bhutta, C. B. (2012). Not by the book: Facebook as a sampling frame. Sociological Methods \& Research, 41(1), 57-88.

Brauer, S. G. (2017). How many congregations are there? Updating a survey-based estimate. Journal for the Scientific Study of Religion, 56(2), 438-448.

Braun, M. T., Kuljanin, G., \& DeShon, R. P. (2018). Special considerations for the acquisition and wrangling of big data. Organizational Research Methods, 21(3), 633-659.

Bright, J., De Sabbata, S., \& Lee, S. (2018). Geodemographic biases in crowdsourced knowledge websites: Do neighbours fill in the blanks? GeoJournal, 83(3), 427-440.

Bryant, E. E., \& DeLozier, J. E. (1968). Developing, maintaining, and evaluating a sampling frame of institutions. Public Health Reports, 83(2), 161-171.

Budhathoki, N. R., \& Haythornthwaite, C. (2013). Motivation for open collaboration: Crowd and community models and the case of OpenStreetMap. American Behavioral Scientist, 57(5), 548-575.

Chaves, M. (2002). Religious organizations: Data resources and research opportunities. American Behavioral Scientist, 45(10), 1523-1549.

Chaves, M., Anderson, S., Eagle, A., Hawkins, M., Holleman, A., \& Roso, J. (2020). National Congregations Study: Cumulative datafile and codebook.

Dennis, J. M. (2019). Technical overview of the AmeriSpeak panel NORC's probability-based household panel. NORC at the University of Chicago.

Firth, D. (1993). Bias reduction of maximum likelihood estimates. Biometrika, 80(1), 27-38.

Fulton, B. R., Bilgen, I., Pineau, V., Liebert, L., King, D. P., \& Dennis, M. (2022). Evaluating nonresponse bias for a hypernetwork sample generated from a probability-based household panel. Journal of Quantitative Methods, (published online before print). 
Fulton, B. R., \& King, D. P. (2018). National Study of Congregations' Economic Practices: Survey Datafile. Indiana University.

Gart, J. J., \& Zweifel, J. R. (1967). On the bias of various estimators of the logit and its variance with application to quantal bioassay. Biometrika, 181-187.

Grønbjerg, K. A., Liu, H. K., \& Pollak, T. H. (2010). Incorporated but not IRS-registered: Exploring the (dark) grey fringes of the nonprofit universe. Nonprofit and Voluntary Sector Quarterly, 39(5), 925-945.

Groves, R. M., Fowler Jr., F. J., Couper, M. P., Lepkowski, J. M., Singer, E., \& Tourangeau, R. (2009). Survey Methodology (Vol. 561). John Wiley \& Sons.

Guharoy, R., Noviasky, J., Haydar, Z., Fakih, M. G., \& Hartman, C. (2013). Compounding pharmacy conundrum: "We cannot live without them but we cannot live with them" according to the present paradigm. Chest, 143(4), 896-900.

Harter, R., Battaglia, M. P., Buskirk, T. D., Dillman, D. A., English, N., Fahimi, M., Frankel, M. R., Kennel, T., McMichael, J. P., \& McPhee, C. B. (2016). Address-Based sampling. Prepared for AAPOR council by the task force on address-based sampling, operating under the auspices of the AAPOR Standards Committee. Oakbrook Terrace, IL. https://www.ncbi.nlm.nih.gov/pubmed/17259351.

Harter, R., Eckman, S., English, N., \& O’Muircheartaigh, C. (Eds.). (2010). Applied sampling for large-scale multi-stage area probability designs.

Hickman, L., Thapa, S., Tay, L., Cao, M., \& Srinivasan, P. (2020). Text Preprocessing for Text Mining in Organizational Research: Review and Recommendations. Organizational Research Methods, (published online before print).

Hirji, K. F., Mehta, C. R., \& Patel, N. R. (1987). Computing distributions for exact logistic regression. Journal of the American Statistical Association, 82(400), 1110-1117.

Ibarz, J., \& Banerjee, S. (2017). Updating Google Maps with deep learning and street view. https://ai.googleblog.com/2017/05/updating-google-maps-with-deep-learning.html

Jarmin, R. S. (2019). Evolving measurement for an evolving economy: thoughts on 21st century US economic statistics. Journal of Economic Perspectives, 33(1), 165-184.

Kalleberg, A. L., Knoke, D., Marsden, P. V., \& Spaeth, J. L. (1994). The National Organizations Study: An introduction and overview. American Behavioral Scientist, 37(7), 860-871.

Kalleberg, A. L., Marsden, P. V., Aldrich, H. E., \& Cassell, J. W. (1990). Comparing organizational sampling frames. Administrative Science Quarterly, 35, 658-688.

Kalton, G. (2021). Introduction to survey sampling. Sage Publications. 
King, D. P., Fulton, B. R., Munn, C. W., \& Goodwin, J. (2019). The National Study of Congregations' Economic Practices Executive Report. Lake Institute on Faith \& Giving.

King, G., \& Zeng, L. C. (2001). Explaining rare events in international relations. International Organization, 55(3), 693-715.

Kobayashi, V. B., Mol, S. T., Berkers, H. A., Kismihók, G., \& Den Hartog, D. N. (2018). Text classification for organizational researchers: A tutorial. Organizational Research Methods, 21(3), 766-799.

Kölln, A.-K., Ongena, Y. P., \& Aarts, K. (2019). The effects of sampling frame designs on nonresponse and coverage error: Evidence from the Netherlands. Journal of Survey Statistics and Methodology, 7(3), 422-439.

Lampkin, L. M., \& Boris, E. T. (2002). Nonprofit organization data: What we have and what we need. American Behavioral Scientist, 45(11), 1675-1715.

Manasse, M. S. (2009). Method for duplicate detection and suppression. US Patent 7,603,370. Google Patents.

McPherson, J. M. (1982). Hypernetwork sampling: Duality and differentiation among voluntary organizations. Social Networks, 3(4), 225-249.

McPherson, M. (2001). Sampling strategies for the arts: A hypernetwork approach. Poetics, 28(4), 291-306.

Murphy, G. B. (2002). The effects of organizational sampling frame selection. Journal of Business Venturing, 17(3), 237-252.

Nemes, S., Jonasson, J. M., Genell, A., \& Steineck, G. (2009). Bias in odds ratios by logistic regression modelling and sample size. BMC Medical Research Methodology, 9(1), 1-5.

Nilsson, I., Reid, N., \& Lehnert, M. (2018). Geographic patterns of craft breweries at the intraurban scale. The Professional Geographer, 70(1), 114-125.

Pitt, R. N. (2021). Church Planters: Inside the World of Religion Entrepreneurs. Oxford University Press.

Popielarz, P. A. (1999). Voluntary association: A multilevel analysis of gender segregation in voluntary organizations. Gender \& Society, 13(2), 234-250.

Rhodes, B. B., Kim, A. E., \& Loomis, B. R. (2015). Vaping the web: Crowdsourcing and web scraping for establishment survey frame generation. Proceedings of the 2015 Federal Committee on Statistical Methodology Research Conference. Available at: https://fcsm.sites.usa.gov/files/2016/03/H3_Rhodes_2015FCSM.,

Samagaio, A., Crespo, N. F., \& Rodrigues, R. (2018). Management control systems in high-tech start-ups: An empirical investigation. Journal of Business Research, 89, 351-360. 
Scheitle, C. P., Dollhopf, E. J., \& McCarthy, J. D. (2016, April 1, 2016). Exploring religious congregations' registration with the IRS. Nonprofit and Voluntary Sector Quarterly, 45(2), 397-408.

Schmidt, M., \& Weiser, P. (2012). Web mapping services: development and trends. In M. P. Peterson (Ed.), Online maps with APIs and web services (pp. 13-21). Springer.

Simsek, Z., \& Veiga, J. F. (2001, July 1, 2001). A primer on internet organizational surveys. Organizational Research Methods, 4(3), 218-235.

Smith, T. W., Davern, M., Freese, J., \& Morgan, S. L. (2019). General Social Surveys, $1972-$ 2018. NORC at the University of Chicago.

Stefanakis, E. (2017). Web Mercator and raster tile maps: Two cornerstones of online map service providers. Geomatica, 71(2), 100-109.

Tonidandel, S., King, E. B., \& Cortina, J. M. (2018). Big data methods:Leveraging modern data analytic techniques to build organizational science. Organizational Research Methods, $21(3), 525-547$.

Tonidandel, S., \& LeBreton, J. M. (2010). Determining the relative importance of predictors in logistic regression: An extension of relative weight analysis. Organizational Research Methods, 13(4), 767-781.

van Smeden, M., Moons, K. G. M., de Groot, J. A. H., Collins, G. S., Altman, D. G., Eijkemans, M. J. C., \& Reitsma, J. B. (2019). Sample size for binary logistic prediction models: beyond events per variable criteria. Statistical Methods in Medical Research, 28(8), 2455-2474.

Weisberg, H. F. (2009). The total survey error approach: A guide to the new science of survey research. University of Chicago Press. 


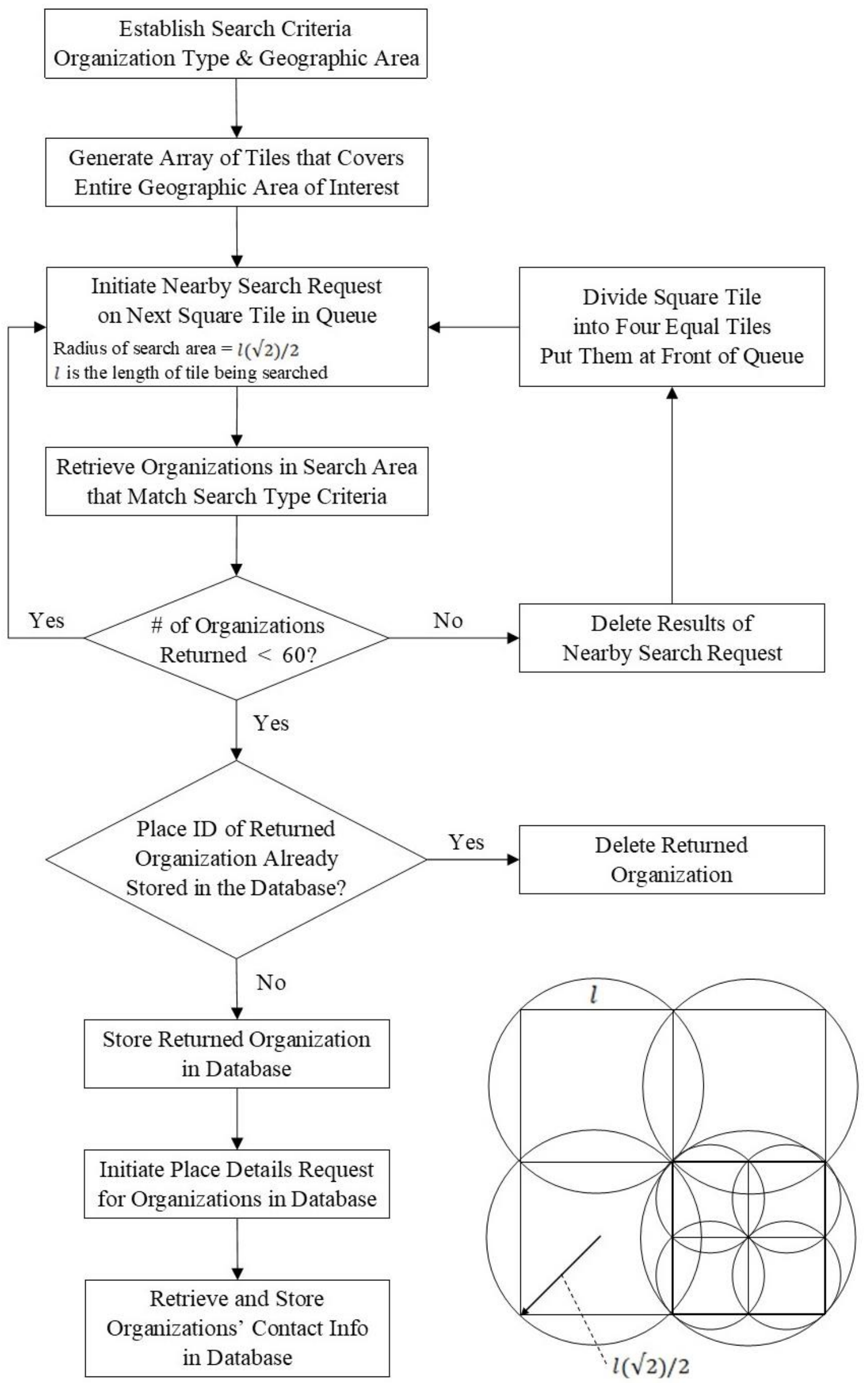

Figure 1. Algorithm for using a raster tile mapping schema to search for particular organizations of interest within specified geographic areas using the Google Maps platform and its Places API. 


\section{Appendix A}

List of hyperlinks to the Google Maps Place API features mentioned in this article

Google Maps Places API: https://developers.google.com/maps/documentation/places/web-service/overview

Place ID: https://developers.google.com/places/web-service/place-id

Place type: https://developers.google.com/places/web-service/supported types

Place details: https://developers.google.com/places/web-service/details

Business categories: https://developers.google.com/my-business/reference/rest/v4/categories

Google My Business: https://www.google.com/business/

Place Search request: https://developers.google.com/places/web-service/search

Nearby Search Request: https://developers.google.com/places/web-service/search\#PlaceSearchRequests

Place Details Request: https://developers.google.com/places/web-service/details\#PlaceDetailsRequests 


\section{Appendix B}

List of Place Types on the Google Maps Platform

(https://developers.google.com/places/web-service/supported_types\#table1)

\begin{tabular}{|c|c|c|}
\hline accounting & fire_station & physiotherapist \\
\hline airport & florist & plumber \\
\hline amusement_park & funeral_home & police \\
\hline aquarium & furniture_store & post_office \\
\hline art_gallery & gas_station & primary_school \\
\hline atm & gym & real_estate_agency \\
\hline bakery & hair_care & restaurant \\
\hline bank & hardware_store & roofing_contractor \\
\hline bar & hindu_temple & rv_park \\
\hline beauty_salon & home_goods_store & school \\
\hline bicycle_store & hospital & secondary_school \\
\hline book_store & insurance_agency & shoe_store \\
\hline bowling_alley & jewelry_store & shopping_mall \\
\hline bus_station & laundry & spa \\
\hline cafe & lawyer & stadium \\
\hline campground & library & storage \\
\hline car_dealer & light_rail_station & store \\
\hline car_rental & liquor_store & subway_station \\
\hline car_repair & local_government_office & supermarket \\
\hline car_wash & locksmith & synagogue \\
\hline casino & lodging & taxi_stand \\
\hline cemetery & meal_delivery & tourist_attraction \\
\hline church & meal_takeaway & train_station \\
\hline city_hall & mosque & transit_station \\
\hline clothing_store & movie_rental & travel_agency \\
\hline convenience_store & movie_theater & university \\
\hline courthouse & moving_company & veterinary_care \\
\hline dentist & museum & zoo \\
\hline department_store & night_club & \\
\hline doctor & painter & \\
\hline drugstore & park & \\
\hline electrician & parking & \\
\hline electronics_store & pet_store & \\
\hline embassy & pharmacy & \\
\hline
\end{tabular}

